\title{
Niobium thin film deposition studies on copper surfaces for superconducting radio frequency cavity applications
}

\author{
W. M. Roach, ${ }^{1, *}$ D. B. Beringer, ${ }^{2}$ J. R. Skuza, ${ }^{3}$ W. A. Oliver, ${ }^{4}$ C. Clavero, ${ }^{1}$ C. E. Reece, ${ }^{5}$ and R. A. Lukaszew ${ }^{1,2}$ \\ ${ }^{1}$ Department of Applied Science, The College of William and Mary, Williamsburg, Virginia 23187, USA \\ ${ }^{2}$ Department of Physics, The College of William and Mary, Williamsburg, Virginia 23187, USA \\ ${ }^{3}$ National Institute of Aerospace, Hampton, Virginia 23666, USA \\ ${ }^{4}$ Department of Mechanical Engineering, Virginia Commonwealth University, Richmond, Virginia 23284, USA \\ ${ }^{5}$ Thomas Jefferson National Accelerator Facility, Newport News, Virginia 23606, USA
}

(Received 29 November 2011; published 26 June 2012)

\begin{abstract}
Thin film coatings have the potential to increase both the thermal efficiency and accelerating gradient in superconducting radio frequency accelerator cavities. However, before this potential can be realized, systematic studies on structure-property correlations in these thin films need to be carried out since the reduced geometry, combined with specific growth parameters, can modify the physical properties of the materials when compared to their bulk form. Here, we present our systematic studies of $\mathrm{Nb}$ thin films deposited onto $\mathrm{Cu}$ surfaces to clarify possible reasons for the limited success that this process exhibited in previous attempts. We compare these films with $\mathrm{Nb}$ grown on other surfaces. In particular, we study the crystal structure and surface morphology and their effect on superconducting properties, such as critical temperature and lower critical field. We found that higher deposition temperature leads to a sharper critical temperature transition, but also to increased roughness indicating that there are competing mechanisms that must be considered for further optimization.
\end{abstract}

DOI: 10.1103/PhysRevSTAB.15.062002

PACS numbers: 74.78.-w, 85.25.Dq, 74.62.Bf, 74.62.Dh

\section{INTRODUCTION}

Superconducting radio frequency (SRF) technology used in particle accelerators is based on bulk $\mathrm{Nb}$ cavities that are approaching the optimal performance achievable with this material [1]. Thus, development of a suitable alternative to bulk $\mathrm{Nb}$ is needed. Because of the very shallow penetration depth of the radio frequency fields, SRF properties are inherently a surface phenomenon involving a material thickness of less than one micron, therefore enabling the possibility of using thin film coatings. The challenge is to understand the dependence of the material's properties on the film deposition parameters and the detailed characteristics of real surfaces and then to employ appropriate techniques to tailor the surface properties for greatest benefit for SRF applications. Several attempts with varying degrees of success have been made in the past to implement thin film $\mathrm{Nb}$ coated $\mathrm{Cu}$ cavities to exploit the better thermal conductivity of $\mathrm{Cu}$ compared to bulk $\mathrm{Nb}$ and also because of the possibility of foreseeing other suitable superconducting coatings [2].

While tight correlation with the characterization of real materials has yet to be described, there exists a theoretical

\footnotetext{
*Corresponding author. wmroach@email.wm.edu

Published by the American Physical Society under the terms of the Creative Commons Attribution 3.0 License. Further distribution of this work must maintain attribution to the author(s) and the published article's title, journal citation, and DOI.
}

framework describing the relevant material parameters as they influence SRF properties. Several material factors contribute to degraded SRF performance with respect to ideal surfaces, such as the presence of intragranular impurities which contributes to a reduction of the electron mean-free path and the local lower critical field $H_{C 1}$. Grain boundaries contribute as scattering centers and may also be a contributing factor to localization of impurities and lossy oxidation states. The presence and diffusion of impurities at grain boundaries and intergranular oxidation contribute "weak links" to the flow of surface supercurrents, creating a nonlinear loss mechanism [3].

In the case of thin films, there are several physical properties that can affect the film's superconducting performance such as surface morphology (roughness), microstructure (grain size/boundaries), and thickness (interfacial scattering due to the substrate being more prominent in thinner films). It is a nontrivial process to separate the individual contributions from each of these factors. However, it is necessary to understand how these factors alter superconducting properties of thin films in order to tailor the materials to achieve optimal performance in SRF cavities.

The first section of this report focuses on $\mathrm{Nb}$ thin films prepared on $\mathrm{MgO}$ surfaces of similar thicknesses in order to determine how the microstructure and surface roughness affect the superconducting properties. Since grain boundaries are an important obstacle to superconducting transport, we show how the increased number of crystallographic domains that can occur during epitaxial $\mathrm{Nb}$ growth in different crystallographic orientations, leading to higher density of grain 
boundaries, can explain some of the superconducting properties observed in these films. Next, we study how the deposition conditions can alter the properties of $\mathrm{Nb}$ thin films on $\mathrm{Cu}$ surfaces. We show our correlated studies of microstructure and surface morphology and the resulting superconducting properties illustrating possible mechanisms responsible for cavity performance when using such films.

\section{EXPERIMENT}

Epitaxial $\mathrm{Nb}$ films have been prepared by DC magnetron sputtering in an ultrahigh vacuum (UHV) system with a base pressure in the low $10^{-10}$ Torr range. This system is a five port Perkin-Elmer molecular beam epitaxy system that has been modified to house sputtering guns. Growth conditions such as geometry, deposition rate, and temperature are remarkably reproducible with the instrument used, thus allowing accurate comparison between samples grown in different runs including thickness series with subnanometer differences between samples [4]. For the present study, the growth was carried out using a 99.95\% pure 2 inch $\mathrm{Nb}$ target with a balanced magnetron at an Ar pressure of 1 mTorr with a target to sample distance was fixed at 7.75 inches, resulting in a growth rate of $0.36 \AA / \mathrm{s}$. These films have been prepared on both $\mathrm{MgO}(100)$ and $\mathrm{Cu}(100)$ surfaces. The ceramic $\mathrm{MgO}$ substrates used in this study were acquired commercially, while the $\mathrm{Cu}$ surfaces were prepared by depositing fresh $\mathrm{Cu}$ layers onto HF-etched $\mathrm{Si}(100)$ in UHV which has been shown to yield smooth $\mathrm{Cu}(100)$ surfaces [5]. The latter process was chosen to avoid native oxides always present on $\mathrm{Cu}$ substrates and surface treatments that might complicate data analysis during our $\mathrm{Nb}$ growth studies. On $\mathrm{MgO}$ substrates, the growth temperature was held at $600^{\circ} \mathrm{C}$ which was found to lead to optimized films, while the $\mathrm{Cu} / \mathrm{Si}$ template temperature was constrained below $175^{\circ} \mathrm{C}$ in order to prevent temperature driven reactions at the interface [6]. The microstructure of the films was studied in situ with reflection high energy electron diffraction (RHEED) which provides a reciprocal space representation of a film's surface. Additional ex situ characterization of film structure was carried out using a four-circle x-ray diffractometer (XRD) with a quasiparallel monochromatic $\mathrm{Cu} K_{\alpha}(\lambda=1.5406 \AA)$ beam, fixed divergence $\left(1 / 4^{\circ}\right)$, antiscattering $\left(1 / 4^{\circ}\right)$, and receiving $\left(1 / 16^{\circ}\right)$ slits, soller slits $(0.04 \mathrm{rad})$, and a proportional detector. The surface morphology of the films was examined ex situ with atomic force microscopy (AFM) and associated software [7]. Superconducting properties were studied using superconducting quantum interference device (SQUID) magnetometry.

\section{NIOBIUM THIN FILMS ON MAGNESIUM OXIDE SURFACES}

\section{A. Microstructure and surface morphology}

$\mathrm{Nb}$ thin films were deposited onto $\mathrm{MgO}(100)$ substrates in order to parametrize the growth conditions and also to characterize the films in an ideal scenario known to yield $\mathrm{Nb}$ films of good crystalline quality before looking at more realistic scenarios. Films presented here range in thickness from 100 to $1000 \mathrm{~nm}$. The epitaxy of the deposited $\mathrm{Nb}$ film is highly dependent on the choice of substrate and initial surface status prior to growth. Our investigations on a significant number of samples indicate that, in the case of the $\mathrm{MgO}(100)$ surface, $\mathrm{Nb}$ films can grow (100) with only one in-plane orientation, $\mathrm{Nb}(100)[011] \| \mathrm{MgO}(100)$ [001], or (110) with two possible in-plane orientations, $\mathrm{Nb}(110) \times$ [ $\left.\begin{array}{lll}1 & 1 & 0\end{array}\right] \mid \mathrm{MgO}(100)[001]$ and $\mathrm{Nb}(110)[001] \| \mathrm{MgO}(100) \times$ [001] depending on the deposition conditions and substrate surface pretreatments. These findings are consistent with previous studies on $\mathrm{Nb}$ films prepared on $\mathrm{MgO}(100)$ [8]. A visual depiction of the possible orientations of $\mathrm{Nb}$ on $\mathrm{MgO}(100)$ is shown in Fig. 1. The typical RHEED patterns associated with the $\mathrm{Nb}$ films agree well with the predicted epitaxy as shown in Fig. 2.

The RHEED images in Fig. 2(a) show regularly spaced sharp streaks along two distinct azimuthal directions which indicate that this film has good crystalline quality with large grain sizes. Figure 2(a) (top) shows streaks that correspond to the $\mathrm{Nb}\langle 110\rangle$ atom spacing while Fig. 2(b) shows streaks that correspond to the $\mathrm{Nb}\langle 100\rangle$ atom spacing agreeing with the previous discussion of $\mathrm{Nb}(100)$ / $\mathrm{MgO}(100)$ epitaxial relationship. The chevron features seen along the $\mathrm{MgO}[100]$ direction are evidence of faceting on the surface, typical features often observed during growth of metallic films exhibiting single crystal structure $[9,10]$. Figure 2(b) also shows sharp streaks, but instead of only one streak spacing, we see a superposition of two different patterns corresponding to atomic spacings with a ratio along the $\mathrm{MgO}[100]$ direction. This observation agrees with the earlier discussion of $\mathrm{Nb}(110) / \mathrm{MgO}(100)$ epitaxy, indicating the coexistence of biaxial equivalent

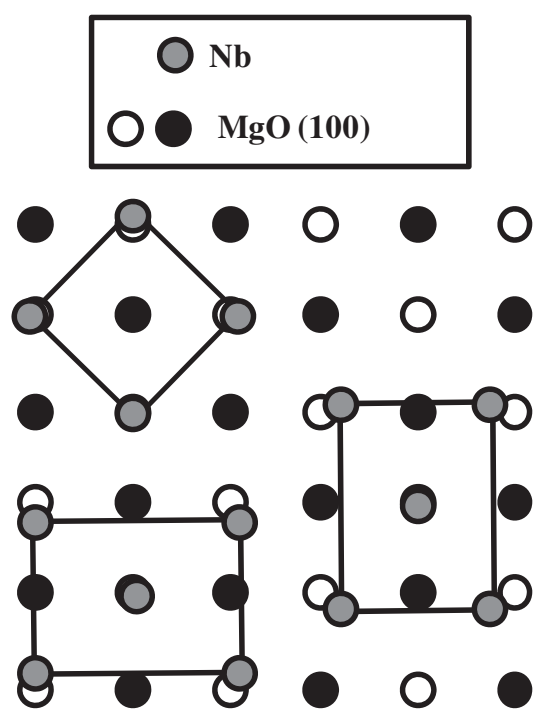

FIG. 1. Overlays of relaxed $\mathrm{Nb}$ lattices on an $\mathrm{MgO}(100)$ surface. 

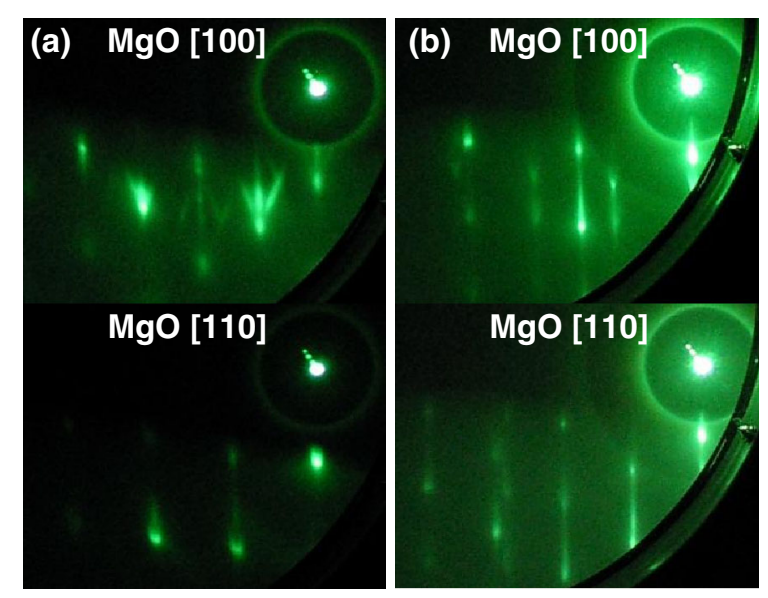

FIG. 2. RHEED patterns for (a) $\mathrm{Nb}(100) / \mathrm{MgO}(100)$ and (b) $\mathrm{Nb}(110) / \mathrm{MgO}(100)$ along the $\mathrm{MgO}[100]$ and $\mathrm{MgO}[110]$ azimuths.

tetragonal structures with two possible in-plane orientations. The pattern observed along the $\mathrm{MgO}$ [110] direction in Fig. 2(b) is similar to contributions from the $\mathrm{Nb}(\overline{1} 11)$ and (112) planes that have been seen in other $\mathrm{Nb}(110)$ growth systems [11].

XRD measurements were carried out ex situ to analyze the out-of-plane lattice parameter and grain size of the thin films. It is worth noticing that, while in situ RHEED characterization is surface sensitive and yields in-plane structural information, XRD probes the entire volume of the film. For all $\mathrm{Nb}$ films investigated that were thicker than $500 \mathrm{~nm}$ in both (100) and (110) orientations, the measured lattice parameter varied less than $1 \%$ from the bulk value of $3.300 \AA$ A. While there was no significant difference between the lattice parameter for films in the 500-1000 nm thickness range considered here, the (100) films consistently had larger grain sizes ranging from $52 \pm 2 \mathrm{~nm}$ to $86 \pm 3 \mathrm{~nm}$, whereas the (110) films had grain sizes around $44 \pm 2 \mathrm{~nm}$. The fact that (100) films exhibit much larger grain sizes than (110) films indicates that smaller grains have coalesced into larger ones as growth proceeded, without generating a boundary (i.e. Ostwald ripening [12]), thus minimizing grain boundary density in this case. This reduction in the overall density of scattering centers for carriers may impact transport measurements. Additionally, the fact that there is no significant change in grain size for (110) films in the 500 to $1000 \mathrm{~nm}$ thickness range indicates that the columnar growth mode leading to the (110) microstructure saturates earlier than in the (100) films since in the latter case coalescence of grains without generation of a new boundary is favored.

A standard measure of merit in superconducting materials is the residual resistance ratio (RRR), which is defined as the ratio of resistances at room temperature (RT) and just above the superconducting transition temperature. For $\mathrm{Nb}, \mathrm{RRR}=R_{300 \mathrm{~K}} / R_{10 \mathrm{~K}}$ is typically used. Resistance in metallic films can be caused by factors such as impurity level [13], film thickness [14], and average grain size [15]. Resistance due to phonons is accounted for by measuring at RT and low temperature, while the resistance due to other scattering mechanisms is temperature independent. Because our films were prepared in identical conditions (background pressure, target purity, growth rate, etc.) and are of comparable thicknesses, we can use RRR values as a relative gauge of grain size or grain boundary density. Consistent with our discussion pertaining to increased grain boundaries in the (110) films, films with thickness ranging from 500 to $1000 \mathrm{~nm}$ exhibited lower RRR values ranging from 26-46.5 when compared to the (100) films with RRR values ranging from 158-165.5 [16].

We note that the nature of the film epitaxy also has a strong effect on the resulting surface morphology as shown in the AFM images shown in Fig. 3. Figure 3(a) shows regular distribution of rounded yet 4-fold symmetric features consistent with (100) single domains found in $\mathrm{Nb}(100) /$ $\mathrm{MgO}(100)$ growth. In the case of (110) films, there are two possible perpendicular domains with uniaxial anisotropy leading to perpendicular uniaxial surface features as shown in Fig. 3(b). The scaling behavior of surface features with increasing film thickness in these two crystallographic orientations has also been examined elsewhere [17].

Since SRF properties are constrained to the surface, and the surface morphology is strongly correlated to the microstructure, understanding how the thin film microstructure affects the superconducting properties is crucial. This is discussed in the following section.

\section{B. Superconducting properties}

The superconducting properties, transition temperature $\left(T_{C}\right)$ and lower critical field $\left(H_{C 1}\right)$, of the films were probed using DC SQUID magnetometry. The transition from the superconducting to nonsuperconducting (or normal) phase occurred around the bulk $T_{C}$ value of $9.2 \mathrm{~K}$ for all the films. The lack of sensitivity of $T_{C}$ to the microstructure indicates that in this case, the grain size of the samples was large enough not to affect this measurement. However, as mentioned earlier, thin films typically have a higher number of defects which can inhibit other superconducting properties [14]. This increase in defect density inhibits

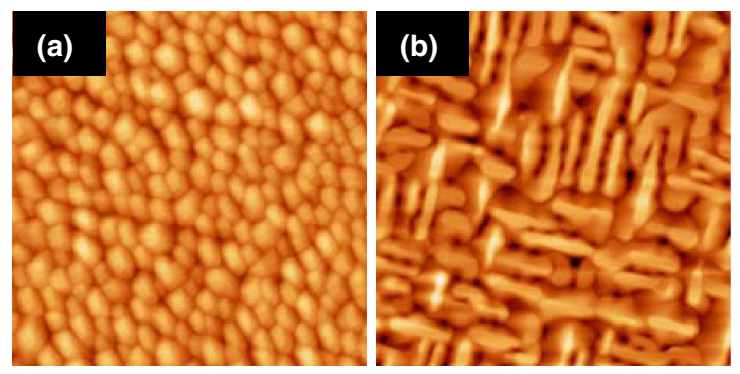

FIG. 3. Representative AFM scans for (a) $\mathrm{Nb}(100)$ and (b) $\mathrm{Nb}(110)$ surfaces. The scan size in both images is $2 \mu \mathrm{m} \times 2 \mu \mathrm{m}$. 
TABLE I. Summary of superconducting properties. $T_{C}$ values were measured with an applied field of $50 \mathrm{Oe} . H_{C 1}$ values were measured at $4 \mathrm{~K}$.

\begin{tabular}{lcccc}
\hline \hline & \multicolumn{2}{c}{$\mathrm{Nb}(100)$} & \multicolumn{2}{c}{$\mathrm{Nb}(110)$} \\
Thickness $(\mathrm{nm})$ & $T_{C}(\mathrm{~K})$ & $H_{C 1}(\mathrm{Oe})$ & $T_{C}(\mathrm{~K})$ & $H_{C 1}(\mathrm{Oe})$ \\
\hline 100 & 9.2 & 1300 & 9.2 & 600 \\
$500-600$ & 9.2 & 1600 & 9.3 & 1200 \\
1000 & 9.3 & 1800 & 9.2 & 1700 \\
\hline \hline
\end{tabular}

entry into the superconducting phase and effectively lowers critical fields in films with comparable thickness.

The procedure presented in Ref. [18] was implemented to determine $H_{C 1}$ values for the $\mathrm{Nb}$ thin films using $\mathrm{DC}$ SQUID magnetometry. When a magnetic field larger than $H_{C 1}$ is applied on a superconducting sample, trapped magnetic fields can be detected by measuring the sample's magnetic moment before and after the application of the field. This procedure begins by cooling the sample with zero applied field to a temperature below the transition temperature, followed by a measurement of the sample's magnetic moment without an applied field. Next, a field is applied parallel to the sample surface and subsequently removed. The procedure continues with another measurement of the sample's magnetic moment with the applied field removed, and then warming the sample above the transition temperature. This method was repeated for many field values. $H_{C 1}$ was then found by determining which applied field first caused a difference in the measurement before the applied field and the measurement after the applied field was removed. When the measurement after the field is removed differs from the measurement before the field is applied, this is an indication that there are trapped fields present in the sample. For this study, $H_{C 1}$ values were measured at $4 \mathrm{~K}$. Values for representative samples are shown in Table I.

For various film thicknesses, the $\mathrm{Nb}(100)$ films consistently have larger $H_{C 1}$ values than their $\mathrm{Nb}(110)$ counterparts suggesting that an increased grain boundary density leads to worse superconducting properties in the latter. While some of the measured $H_{C 1}$ values are larger than values that others have measured in bulk samples [19], a recent study by Roy et al. suggests that the field at which flux lines begin to penetrate can be higher than previously reported values [20]. The field value at which flux lines begin to penetrate is highly dependent on the shape of the sample as well as the condition of the surface. For their studies, Roy et al. prepared small samples of large grain $(1 \mathrm{~mm}) \mathrm{Nb}$ and carried out isothermal magnetization versus field measurements. For these small, high quality samples, a penetration field of at least $2000 \mathrm{Oe}$ at $3 \mathrm{~K}$ was found.

\section{NIOBIUM THIN FILMS ON COPPER SURFACES}

As mentioned above, it is desirable to use $\mathrm{Nb}$ coated $\mathrm{Cu}$ cavities to improve their thermal efficiency for SRF applications, but it is necessary to understand how the preparation of such cavities will affect their overall performance. To this end, we investigated how the deposition temperature can affect the structure and superconducting properties on $\mathrm{Cu}$ surfaces. Thus, $500 \mathrm{~nm}$ thick epitaxial $\mathrm{Cu}$ films were deposited onto $\mathrm{Si}(100)$. Subsequently, $500 \mathrm{~nm}$ $\mathrm{Nb}$ films were deposited onto the $\mathrm{Cu}(100)$ template under identical conditions as the films in Sec. III with the exception that some films were deposited at RT while others were deposited at a substrate temperature of $150^{\circ} \mathrm{C}$.

\section{A. Microstructure and surface morphology}

To interpret our results we need to first discuss the possible epitaxial relationships that $\mathrm{Nb}$ can follow when deposited onto a $\mathrm{Cu}(100)$ surface. In this case, there are four possible in-plane orientations such that the out-ofplane orientation is $\mathrm{Nb}(110)[111] \| \mathrm{Cu}(100)[110]$ [11]. A visual depiction of the possible orientations of $\mathrm{Nb}$ on $\mathrm{Cu}(100)$ is shown in Fig. 4. Our in situ RHEED characterization agrees with the expected epitaxy as shown in Fig. 5(a). Figure 5(a) contains broader streaks than those seen in Fig. 2, indicating a smaller lateral grain size when $\mathrm{Nb}$ is deposited on $\mathrm{Cu}$ versus $\mathrm{MgO}$. Again, we see a superposition of two patterns with a $\sqrt{2}$ along the $\mathrm{Si}$ [100] direction indicating the formation of orthogonal $\mathrm{Nb}(110)$ domains. The patterns seen along the $\mathrm{Si}[110]$ direction correspond to contributions from the $\mathrm{Nb}(\overline{1} 11)$ and $(\overline{1} 12)$ planes as mentioned earlier [11].

The surface morphology of $\mathrm{Nb}$ films grown on $\mathrm{Cu}$ displayed features similar to those seen in the $\mathrm{Nb}(110) /$ $\mathrm{MgO}(100)$ samples as shown in Fig. 5(b). The surface features on samples prepared at RT were finer than those found on samples prepared at $150^{\circ} \mathrm{C}$ and resulted in a

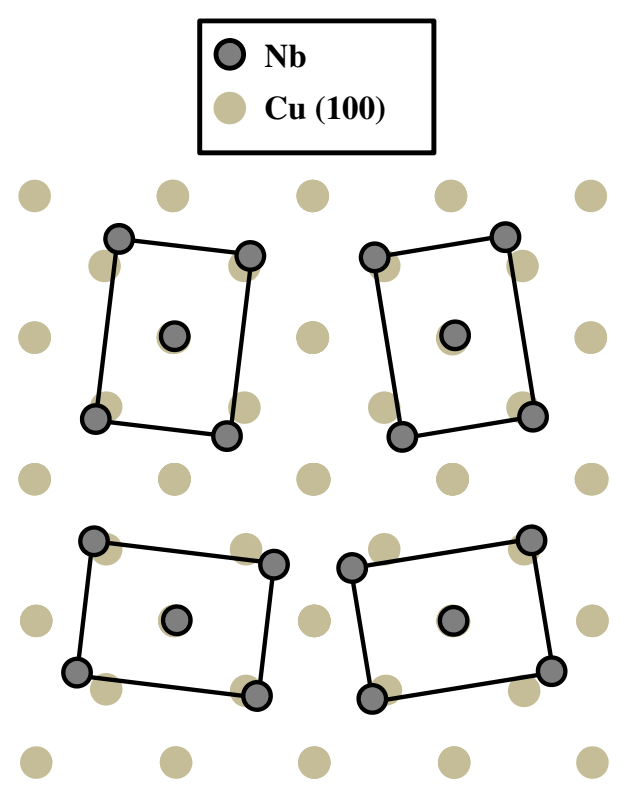

FIG. 4. Overlays of relaxed $\mathrm{Nb}$ lattices on a $\mathrm{Cu}(100)$ surface. 


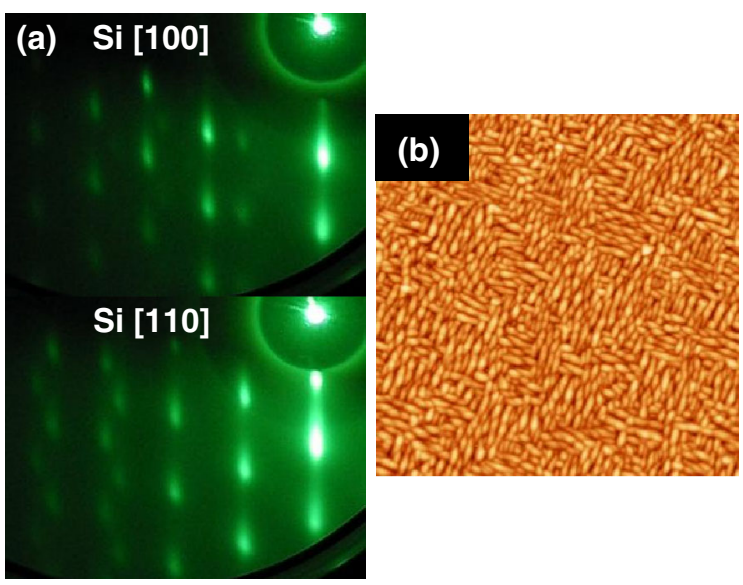

FIG. 5. (a) RHEED pattern for $\mathrm{Nb}(110) / \mathrm{Cu}(100) / \mathrm{Si}(100)$ along the $\mathrm{Si}[100]$ and $\mathrm{Si}[110]$ azimuths. (b) A representative $2 \mu \mathrm{m} \times 2 \mu \mathrm{m}$ AFM scan for $\mathrm{Nb}$ films on the $\mathrm{Cu}$ template.

lower root mean square (RMS) roughness $(1.98 \mathrm{~nm}$ for RT, $2.87 \mathrm{~nm}$ for $150^{\circ} \mathrm{C}$ ).

XRD measurements on these films give a lattice parameter exhibiting less than $1 \%$ strain compared to the bulk value, similar to the previous case of $\mathrm{Nb}$ growth on $\mathrm{MgO}$. For the growth carried out at RT, the typical average grain size was found to be $44 \pm 2 \mathrm{~nm}$ while for growth at $150^{\circ} \mathrm{C}$ it was $50 \pm 2 \mathrm{~nm}$. Clearly, the increased growth temperature allowed for the formation of larger grains.

\section{B. Superconducting properties}

While films grown at both RT and $150^{\circ} \mathrm{C}$ had transition temperatures of about $9.2 \mathrm{~K}$, the sharpness of the transition is quite different as seen in Fig. 6. The films grown at $150^{\circ} \mathrm{C}$ have a very sharp transition from the superconducting state to the normal state that begins at $\sim 9 \mathrm{~K}$ while films grown at RT have a much more gradual transition. The RT sample's response in the superconducting state begins to decrease as low as $7 \mathrm{~K}$ before entering the normal state.

$H_{C 1}$ values for these films were measured at $4 \mathrm{~K}$ using the method previously described using 10 Oe increments. For RT growth, an $H_{C 1}$ value of 50 Oe was found while an $H_{C 1}$ value of 100 Oe was found for growth at $150^{\circ} \mathrm{C}$. We expect that four possible orientations are more detrimental than two orientations in terms of crystalline film quality as this could lead to smaller lateral grains, even if XRD shows similar out-of-plane grain sizes. Our findings are similar to other reports of sputtered $\mathrm{Nb}$ films, such as Antoine who finds an $H_{C 1}$ value of $180 \mathrm{Oe}$ for $250 \mathrm{~nm} \mathrm{Nb} / \mathrm{Al}_{2} \mathrm{O}_{3}(1 \overline{1} 20)$ ( $r$-plane sapphire) [21]. While this epitaxy may yield one orientation of $\mathrm{Nb}$ [22] (and therefore fewer grain boundaries), it is highly likely that the low thickness decreases the $H_{C 1}$ value due to interfacial scattering compared to thicker films and is therefore a dominant factor here.

Our results suggest that an increased deposition temperature of $\mathrm{Nb}$ onto $\mathrm{Cu}$ leads to films with higher crystalline quality (grain size) and thus improved super-

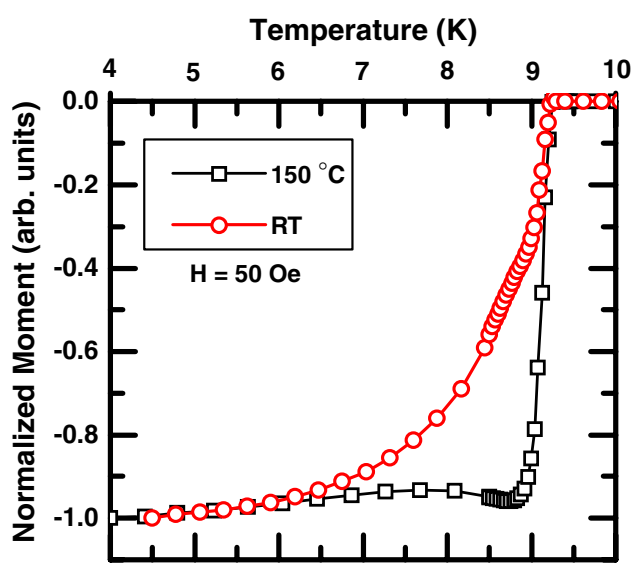

FIG. 6. Critical temperature transitions for films deposited at RT and $150^{\circ} \mathrm{C}$.

conducting properties $\left(H_{C 1}\right)$. Additionally, the increased deposition temperature improves the sharpness of the transition from the superconducting state into the normal state. Therefore, when $\mathrm{Nb}$ coated $\mathrm{Cu}$ cavities are fabricated, our studies suggest that their performance would benefit if they are held at an elevated temperature during the deposition of $\mathrm{Nb}$ coatings.

\section{CONCLUSIONS AND OUTLOOK}

To understand the main mechanisms affecting superconducting $\mathrm{Nb}$ films we investigated first $\mathrm{Nb}$ growth onto ceramic substrates, which leads to well controlled epitaxy. Thus, we found that in order to achieve $\mathrm{Nb}$ coated $\mathrm{Cu}$ cavities with optimal performance, it may be necessary to minimize multiple domain growth and hence decrease grain boundary density in epitaxial $\mathrm{Nb}$ that in principle leads to four domains on $\mathrm{Cu}(100)$ and six domains on $\mathrm{Cu}(111)$ surfaces. We investigated the effect of deposition temperature on the superconducting properties of $\mathrm{Nb}$ films grown on $\mathrm{Cu}$ surfaces and found that higher deposition temperature leads to a sharper critical temperature transition, but also to increased surface roughness. In order to successfully implement $\mathrm{Nb}$ coated $\mathrm{Cu}$ cavities, the coating parameters need to be optimized such that both the surface and superconducting properties lead to the desired SRF performance. To overcome some of the observed difficulties, we have explored the use of a seed layer material such as Au, placed between the $\mathrm{Cu}$ surface and the $\mathrm{Nb}$ film. We were able to achieve epitaxial growth and decreased surface roughness. We note that the process of minimizing grain boundary density still allows for further optimization, and other seed layer materials are being considered to this effect.

\section{ACKNOWLEDGMENTS}

This work was funded by the Defense Threat Reduction Agency (HDTRA1-10-1-0072) and the U.S. Department of Energy (DE-AC05-06OR23177). 
[1] P. Kneisel, G. R. Myneni, G. Ciovati, J. Sekutowicz, and T. Carneiro, in Proceedings of the 21st Particle Accelerator Conference, Knoxville, 2005 (IEEE, Piscataway, NJ, 2005), ТPPT076.

[2] S. Calatroni, Physica C 441, 95 (2006).

[3] R. Russo, Meas. Sci. Technol. 18, 2299 (2007).

[4] C. Clavero, K. Yang, J. R. Skuza, and R. A. Lukaszew, Opt. Express18, 7743 (2010).

[5] C.-A. Chang, J. C. Liu, and J. Angillello, Appl. Phys. Lett. 57, 2239 (1990).

[6] L. Stolt, A. Charai, F. M. D'Heurle, P. M. Fryer, and J. M. E. Harper, J. Vac. Sci. Technol. A 9, 1501 (1991).

[7] I. Horcas, R. Fernandez, J. M. Gomez-Rodriguez, J. Colchero, J. Gomez-Herrero, and A. M. Baro, Rev. Sci. Instrum. 78, 013705 (2007).

[8] Y. Igarashi and M. Kanayama, J. Appl. Phys. 57, 849 (1985).

[9] H. R. Gutiérrez, M. A. Cotta, and M. M. G. de Carvalho, Appl. Phys. Lett. 79, 3854 (2001).

[10] K. Pelhos. T. E. Madey, J. B. Hannon, and G. L. Kellogg, Surf. Rev. Lett. 6, 767 (1999).

[11] K. Mašek and V. Matolin, Vacuum 61, 217 (2001).

[12] R. El-Khozondar, H. El-Khozondar, G. Gottstein, and A. Rollet, Egypt. J. Solids 29, 35 (2006)

[13] J. Sosniak and G. W. Hill, J. Appl. Phys. 38, 4390 (1967).
[14] A. F. Mayadas, R. B. Laibowitz, and J. J. Cuomo, J. Appl. Phys. 43, 1287 (1972).

[15] A.F. Mayadas and M Shatzkes, Phys. Rev. B 1, 1382 (1970).

[16] We note that interfacial scattering can also affect the RRR values and therefore thicker films of good crystalline quality can exhibit even larger RRR values. In fact, larger RRR values have been reported for thicker $\mathrm{Nb}$ films epitaxially grown on $\mathrm{MgO}(100)$. See M. Krishnan, E. Valderrama, B. Bures, K. Wilson-Elliot, X. Zhao, L. Phillips, A.-M. Valente-Feliciano, J. Spradlin, C. Reece, and K. Seo, Supercond. Sci. Technol. 24, 115002 (2011).

[17] D. B. Beringer, W. M. Roach, C. Clavero, C. E. Reece, and R. A. Lukaszew, Phys. Rev. ST Accel. Beams (to be published).

[18] C. Böhmer, G. Brandstätter, and H. W. Weber, Supercond. Sci. Technol. 10, A1 (1997).

[19] D. K. Finnemore, T.F. Stromberg, and C. A. Swenson, Phys. Rev. 149, 231 (1966).

[20] S. B. Roy, G. R. Myneni, and V. C. Sahni, Supercond. Sci. Technol. 21, 065002 (2008).

[21] C.Z. Antoine, S. Berry, M. Aurino, J.-F. Jacquot, J.-C. Villegier, G. Lamura, and A. Andreone, IEEE Trans. Appl. Supercond. 21, 2601 (2011).

[22] A. R. Wildes, J. Mayer, and K. Theis-Bröhl, Thin Solid Films 401, 7 (2001). 